\title{
Context-Aware Rate-Adaptive Beaconing for Efficient and Scalable Vehicular Safety Communication
}

\author{
Alvin Sebastian, Maolin Tang, Yanming Feng, Mark Looi \\ School of Electrical Engineering and Computer Science, Queensland University of Technology, Brisbane, Australia \\ Email: a.sebastian@qut.edu.au,m.tang@qut.edu.au,y.feng@qut.edu.au,m.looi@qut.edu.au
}

Received May 31, 2012; revised July 11, 2012; accepted August 14, 2012

\begin{abstract}
Vehicular safety applications, such as cooperative collision warning systems, rely on beaconing to provide situational awareness that is needed to predict and therefore to avoid possible collisions. Beaconing is the continual exchange of vehicle motion-state information, such as position, speed, and heading, which enables each vehicle to track its neighboring vehicles in real time. This work presents a context-aware adaptive beaconing scheme that dynamically adapts the beaconing repetition rate based on an estimated channel load and the danger severity of the interactions among vehicles. The safety, efficiency, and scalability of the new scheme is evaluated by simulating vehicle collisions caused by inattentive drivers under various road traffic densities. Simulation results show that the new scheme is more efficient and scalable, and is able to improve safety better than the existing non-adaptive and adaptive rate schemes.
\end{abstract}

Keywords: VANET; DSRC; Vehicular Safety Communication; Safety Applications; Adaptive Beaconing; Context-Aware

\section{Introduction}

Recent advances in wireless communication technology have resulted in the development of a Cooperative Collision Warning System (CCWS) that can actively prevent accidents, and therefore may improve road safety significantly. Several concepts and prototypes of the CCWS have been proposed and developed [1-3], demonstrating the technical feasibility of the CCWS. The CCWS works by having vehicles to continually exchange safety messages via wireless ad hoc networks. The safety messages, termed as beacon messages, contain up-to-date vehicle state information, such as position, speed, heading, and other kinematics or motion information. The dissemination of beacon messages, termed as beaconing, allows each vehicle to realize and track the existence and the state information of its neighboring vehicles within a certain range. Using the state information, each vehicle can predict any possible collision and provide early warnings to its driver accordingly.

The wireless technology used in the CCWS will be based on the IEEE $802.11 \mathrm{p}$ [4] and the IEEE 1609 Wireless Access in Vehicular Environments (WAVE) [5] standards. Extensive studies on the performance of the standards $[6,7]$ indicate that the standards can provide an adequate signal reception in an environment with highspeed mobility. However, the standard alone cannot ensure time-critical message dissemination in dense road traffic conditions, such as in traffic jams. Dense traffic conditions induce a high communication channel load, which causes a higher rate of packet collisions and significantly deteriorates the communication performance [6]. To ensure fast and reliable delivery of beacon messages to all relevant vehicles in any traffic conditions, it is necessary to develop application level protocols that can utilize the communication channel more efficiently.

Typical CCWS and other safety applications assume that a vehicle broadcasts beacon messages periodically at a constant rate of ten messages per second [8]. The constant rate beaconing strategy is simple and easy to implement, but is not scalable to various road traffic situations. Road traffic is a very dynamic environment, in which the vehicle density can vary significantly over time. If the broadcast rate and other parameters such as radio range and packet size are constant, the communication performance can vary depending on the vehicle density. A dense traffic condition may lead to a high rate of packet loss that can compromise the safety performance of the CCWS significantly. Therefore, to reduce channel congestion and improve communication performance, the beaconing rate should be continuously adapted to the traffic situation $[9,10]$. Existing rate-adaptive beaconing schemes [11-13] are designed to improve mainly the communication performance. However, they do not consider the differences in the danger severity of an interacttion between two vehicles that may lead to a possible collision. By prioritizing vehicles based on their danger severity, it may be possible to further improve the safety performance. 
In this article, we propose a new context-aware beaconing scheme that considers the danger severity of vehicle interactions in reducing the beaconing rate. For example, Figure 1 shows a simple traffic situation where vehicles $v_{1}, v_{2}$, and $v_{3}$ are following each other with an unsafe following distance while vehicles $v_{4}$ and $v_{5}$ are moving independently. Assuming a high channel usage in the vicinity, each vehicle needs to cooperatively reduce their beaconing rate. Because of the unsafe conditions, reducing the beaconing rate of vehicle $v_{1}$ may significantly increase the possibility of collisions with vehicles $v_{2}$ and $v_{3}$. In contrast, reducing the beaconing rate of vehicle $v_{5}$ will not significantly increase the possibility of collisions between $v_{5}$ and other vehicles. Vehicles that endanger other vehicles such as $v_{1}$ and $v_{2}$ should have a higher beaconing rate compared to vehicles that are unlikely to endanger other vehicles, such as $v_{4}$ and $v_{5}$.

The original contribution of this work is a new beaconing scheme that continuously adapts the beaconing rate to the estimated channel load and the danger severity of the interactions among vehicles. The objective of this research is to optimize the beaconing rate of each vehicle in order to improve the capability of the CCWS collision prevention in various traffic conditions. The improvement is achieved by controlling channel usage to avoid congestion, and most importantly, by prioritizing the most endangered vehicles. The performance of the new scheme is evaluated by simulating vehicle collisions caused by inattentive drivers. Simulation results show that the adaptive rate scheme consistently provides a better safety level on highways in various traffic densities compared to the existing constant and adaptive rate schemes.

The rest of this article is organized as follows: Section 2 introduces the related work and identifies the knowledge gap in the literature. Section 3 presents a new context-aware scheme for beaconing rate adaptation. The performance of the proposed scheme is evaluated in Section 4. Section 5 discusses and analyzes the experimental results. Finally, Section 6 concludes this work and proposes future research direction.

\section{Related Work}

The IEEE $802.11 \mathrm{p}$ WAVE standards [4,5] define a single control channel to be used exclusively for all safety

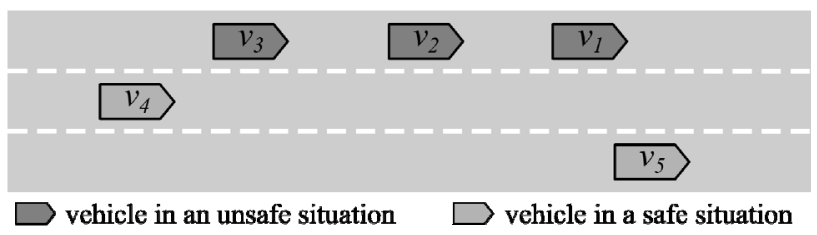

Figure 1. Example of a simple traffic situation with differing danger severity between vehicles. related communication, which includes beaconing. Since the channel is shared by all communication nodes, beaconing may saturate the channel bandwidth in a dense traffic situation. To ensure safety, the beacon messages must be prevented from overloading the control channel. In literature, there are two categories of schemes that have been proposed to improve the performance of beaconing: schemes that increase the effective capacity, and schemes that control the beaconing load.

The effective capacity can be increased by controlling the transmission timing to reduce the possibility of packet collisions, improve reception rate, and ensure fairness to channel access time. An example of this approach is a collision-free scheduling of packet transmissions into time slots [14]. Controlling the transmission timing does not prevent channel congestion as it does not reduce or limit the actual beaconing load generated by vehicles. Therefore, it is not the main solution to ensure the function of safety application.

The beaconing load can be controlled by tuning various parameters that contribute to the communication density. Communication density is described as the product of vehicle density, message size, message generation rate, and transmission range [15]. Since vehicle density is determined based on the actual road traffic conditions, only the three other parameters can be optimized: message size, message generation rate, and transmission range. Several studies on the effect of these parameters to network performance $[9,10,16]$ indicate the need for adaptive algorithms to control the channel load by adjusting the parameters dynamically based on the surrounding traffic conditions.

The message size can be reduced by utilizing a message dispatcher to control all data exchanges between applications and prevent the same elements from being transmitted multiple times by different applications [17]. The transmission range can be reduced by adjusting the per-packet transmission power based on the estimated vehicle density on the road $[18,19]$. The message generation rate (beaconing rate) parameter is the main focus in this article. In contrast to the other two parameters, the message generation rate directly affects the CCWS safety performance. Therefore, the beaconing rate should be minimized without reducing tracking accuracy and compromising safety.

Existing studies of adaptive beaconing rate schemes use the metric of tracking accuracy to measure the safety performance. Their goal is to reduce or control the beaconing rate while maintaining a sufficient level of tracking accuracy. Rezaei et al. [20] presented a scheme to adapt the beaconing rate depending on a position predicttion error. Since the movement of a vehicle is predictable to some degree, a beacon message needs to be sent only when the prediction error is greater than a specified error 
threshold. For example, a prediction error can be caused by a relatively noticeable change of course such as acceleration or a change of direction. Armaghan et al. [21] further improved the idea by dynamically adapting the error threshold and the number of estimation steps based on safety distance. Each vehicle estimates its location ahead for several intervals and sends the information along with its actual current position. While the estimated information is available, there is no new transmission unless any estimation errors are detected. Note that the defined maximum error can actually be exceeded due to message loss. Considering the dynamic nature of traffic density, it is possible that even the reduced beaconing rate is still relatively high enough to cause channel congestion. For example, on a wide highway with many lanes, a traffic jam will cause frequent occurrences of a sudden change of movement (stop and go situation) that will result in many beacon messages being sent frequently.

To maintain a consistent beaconing performance in all traffic situations with varying density, the actual or estimated channel load must be considered. In principle, beaconing rate should be reduced when the channel load becomes higher. Saito et al. [11] proposed a scheme that can estimate the channel load based on the number of reception messages and detected reception errors, and adapt the beaconing rate accordingly. Huang et al. [22] proposed a similar scheme that calculates a transmission probability based on the estimated tracking error. The tracking error is stochastically decided depending on the estimated channel load. The approach had been shown to be more scalable to various vehicle densities and can be complemented with a simple adaptive transmission range scheme [12].

All the aforementioned adaptive schemes are unable to prioritize the vehicles that are in a more dangerous situation than vehicles in a relatively safer situation. To address this problem, we propose a new scheme that uses the vehicle interaction graph [23] to prioritize vehicles in the most danger and improve both the communication and safety performance of the CCWS. Since the tracking accuracy required in preventing a collision depends relatively to interactions among vehicles, safety performance is evaluated by simulating the number of potential collisions.

\section{Beaconing Rate Adaptation Using Context Information}

Every vehicle repeatedly sends a beacon message to all other vehicles with an interval $I$ between two consecutive transmissions. The beaconing interval $I$ directly determines the beaconing rate, which is the number of messages sent per second. The interval $I$ can be predetermined as a constant for all time or can be determined dynamically in real time.

A vehicle $v_{1}$ that receives a beacon message sent by other vehicle $v_{2}$ at time $t_{1}$ knows the state of vehicle $v_{2}$ at time $t_{1}$. Tracking accuracy is defined as the difference between the state of vehicle $v_{2}$ at time $t_{1}$ as tracked by vehicle $v_{1}$ and the actual state of vehicle $v_{2}$ at current time $t_{\text {now }}=t_{1}+\Delta t$. The most relevant metric for tracking accuracy is positional distance error [12,20], which measures the distance between the tracked position and the actual position. Generally, the tracking accuracy is higher if the duration $\Delta t$ is shorter, which can be achieved by increasing the reception rate of beacon messages. Since the communication channel capacity is limited, the reception rate cannot be increased indefinitely by increasing the beaconing rate, which limits the achievable tracking accuracy.

Higher tracking accuracy will result in a more accurate collision prediction [1,24]. As an inaccurate prediction may lead to a collision, tracking accuracy has been used as an indicator to assess the safety performance of beaconing schemes. However, the possibility of a collision mostly depends on the danger severity of a vehicle's traffic situation. For example, consider a vehicle $v_{s}$ that is in a safe situation as opposed to another vehicle $v_{u}$ that is in an unsafe situation. Vehicle $v_{s}$ has a smaller possibility of being involved in a collision, and therefore vehicle $v_{s}$ does not require its warning or tracking accuracy to be as high as of vehicle $v_{u}$. One vehicle at a particular time may not require the same tracking accuracy as another vehicle to maintain the same safety performance.

Danger severity is determined from a time duration $\tau$ that is available to perform an evasive action before a collision becomes unavoidable, given a particular interaction between vehicles. A longer duration $\tau$ provides a driver more time and opportunity to react and avoid a possible collision. To optimize channel usage without compromising safety, the beaconing interval $I$ must be made adaptive based on an estimated load and the danger severity. The beaconing interval $I$ of a vehicle can be proportionally adjusted based on the ratio between the danger severity of itself and of its neighboring vehicles. The basic principle is to give the highest priority (shortest interval) to vehicles in the most danger to avoid any possible collisions and at the same time control the channel load.

\subsection{System Assumptions}

This work assumes that every vehicle is equipped with a wireless communication device that complies to the IEEE $802.11 \mathrm{p}$ WAVE standards, which define the protocols for PHY, MAC, and network layers. All the communication devices operate in ad hoc mode and there is no roadside infrastructure available. A vehicle is assumed to be able to determine its own position on the 
road, with an accuracy suitable for safety purposes, using a combination of a Differential Global Positioning System (DGPS) and internal motion sensors [1,2,25].

\subsection{Problem Definition}

Let $v$ be a subject vehicle and $V^{\prime}$ be a set of vehicles within one-hop communication range of $v$. The state of vehicle is defined as a tuple $(x, y, w, l, \theta, s, \alpha)$, where $x$ and $y$ are the position coordinates, $w$ is the width, $l$ is the length, $\theta$ is the heading, $s$ is the speed, and $\alpha$ is the maximum deceleration of the vehicle. The identity and state of each vehicle $u \in V^{\prime}$ are obtained from a received beacon message. The state of the subject vehicle $v$ is obtained from its internal positioning system. A set of vehicles $V=\{v\}$ $\cup V^{\prime}$ is maintained locally by every subject vehicle $v$.

Given a constant message size $S$ and the physical data rate of wireless communication $R$, a transmission duration for a single message $T=S / R$ can be calculated. The duration $T$ excludes the extra time taken by PHY or MAC protocol overhead. For example, a single transmission of a message with a size of 500 bytes using a data rate of $6 \mathrm{Mbps}$ will occupy the communication channel for 0.6 milliseconds. The channel load $\lambda$ can be estimated based on the number of nodes in the transmission range of each other $n$, the message generation rate of each node $f_{i}$, and the transmission duration $T$ :

$$
\lambda=\sum_{i=1}^{n} f_{i} \cdot T
$$

For example, given the number of nodes $n=50$, the same generation rate for each node $f=10 \mathrm{~Hz}$, and transmission duration $T=0.0006 \mathrm{~s}$, the channel load will be: $\lambda$ $=50 \times 10 \times 0.0006=0.3$. In the CSMA-based protocol such as IEEE $802.11 \mathrm{p}$, the channel becomes more congested as $\lambda$ approaches 1 . To control the channel load consumed by beaconing, the beaconing rate $f$ for each vehicle must be adapted contextually. At each point in time, the rate $f$ may differ for each vehicle as each vehicle may have different neighboring vehicle density and danger severity (i.e., the context information).

Therefore, the problem of adaptive beaconing rate can be defined as follows:

- Input: The set of vehicles $V$, the transmission duration of a single beacon message $T$, and the maximum beaconing load $\lambda_{\max }$ as a parameter to control channel utilization.

- Output: The beaconing interval $I$ or rate $f=1 / I$ for each subject vehicle such that $\lambda_{\max }$ is not exceeded and the most endangered vehicles are assigned with the shortest interval.

\subsection{Multi-Vehicle Interaction Graph}

The danger severity of vehicles in a certain proximity is estimated by finding interactions among multiple vehi- cles. Previously, we have proposed a multi-vehicle interaction graph model [23] to represent the interaction between multiple vehicles in a specific region and at a point in time. The original model has been extended to include the calculation of the danger severity.

The vehicle interaction graph is defined as a weighted directed graph $G=(V, E)$, where $V$ represents the vehicles in a specific area and $E$ represents the interactions among vehicles. An edge $e_{i j} \in E$ represents an interaction between vehicles $i$ and $j$, where vehicle $i$ is influencing vehicle $j$. An edge weight $\omega_{i j}, 0<\omega_{i j} \leq 1$ is a real number that indicates the danger severity or intensity of the interaction $e_{i j}$. The value of $\omega_{i j}=1$ indicates an interaction with the highest severity while $\omega_{i j}=0$ indicates no interaction.

Each vehicle maintains its own interaction graph. Given the set of vehicles $V$ tracked by each subject vehicle, an interaction graph $G$ is constructed by generating the set of edges $E$ and the corresponding edge weight. Initially, each vehicle $v$ creates a graph $G=(V, E)$, where $V=\{v\}$ and $E=\varnothing$. Every time a vehicle $v$ receives a beacon message from other vehicle $v_{i} \in V^{\prime}$, vehicle $v$ updates its interaction graph $G$ by enumerating each vehicle $v_{j} \in V$. For each pair $\left(v_{i}, v_{j}\right), i \neq j$, the interactions between $v_{i}$ and $v_{j}$ are calculated based on their position, speed, and heading. Depending on the result, an edge $e_{i j}$, $e_{j i}$, or both edges, may be added to the set of edges $E$. As an example, Figure 2 shows a possible interaction graph that represents the traffic situation shown in Figure 1.

An interaction is determined if there is a trajectory contention between a pair of vehicles and their avoidance time $\tau$ is less than or equal to the maximum reaction time $T_{\max }$. The avoidance time $\tau$ is the time available for the driver of the influenced vehicle to react in order to avoid the collision. The maximum reaction time $T_{\max }$ is a parameter that reflects the worst possible reaction time for a driver. The minimum reaction time $T_{\min }$ reflects the best possible reaction time for a driver. The danger severity, represented as an edge weight $\omega$, is determined based on the value of $\tau$ scaled proportionally with $T_{\min }$ and $T_{\max }$. Any interaction with an avoidance time less than $T_{\text {min }}$ will be treated as having the same severity. Based on the statistics [26], this study assumes the value of $T_{\min }=0.2 \mathrm{~s}$ and $T_{\max }=2.5 \mathrm{~s}$. The following equations are used to calculate the danger severity:

$$
\begin{gathered}
\tau=\max \left(T_{\min }, \tau\right) \\
\omega(\tau)=1-\frac{\tau-T_{\min }}{T_{\max }-T_{\min }} \\
\text { (24) (12) }
\end{gathered}
$$

Figure 2. Example of an interaction graph that represents the traffic situation shown Figure 1. 
Trajectory contention is calculated by considering three distinct cases covering all the possible traffic scenarios without road information from a digital map. Depending on the case, the avoidance time $\tau$ is calculated differently based on their vector geometry and kinematic calculations. For the sake of simplicity, the absolute position of vehicle $v$ is represented by a Cartesian coordinate $\left(x_{v}, y_{v}\right)$, referenced as the center point of the vehicle. The heading of the vehicle $\theta_{v}$ is in radian where $0 \leq \theta_{v}<$ $2 \pi$, and $\theta_{v}=0$ means heading north. Let $A$ and $B$ be a pair of vehicles to be processed.

Following: This is a case where one vehicle is following another vehicle (vehicle $A$ is following $B$ or vice versa). This case applies if vehicles $A$ and $B$ are moving in the same direction and have overlapping paths. After determining the following vehicle $F$ and the leading vehicle $L$, the net distance $d_{t}$ can be calculated using Equation (4), where $d_{a}$ is the actual longitudinal distance between vehicles $F$ and $L, d_{\min }$ is a parameter of the expected minimal distance between vehicles, and $l_{F}$ and $l_{L}$ are the length of vehicles $F$ and $L$, respectively.

$$
d_{t}=d_{a}-d_{\min }-\frac{1}{2}\left(l_{F}+l_{L}\right)
$$

There are two conditions in this case that can lead to a possible collision. First, the follower is faster than the leader. Second, the distance between them is less than the safety distance. The second condition is used to anticipate an event when the leader brakes abruptly. The conditions are modeled using a different avoidance time $\tau_{1}$ and $\tau_{1}$ :

$$
\begin{aligned}
& \dot{\tau}_{1}=\frac{d_{t}}{s_{F}-s_{L}}-\frac{s_{F}-s_{L}}{2 \alpha_{F}} \\
& \ddot{\tau}_{1}=\frac{d_{t}}{s_{F}}+\frac{1}{2 s_{F}}\left(\frac{s_{L}^{2}}{\alpha_{L}}-\frac{s_{F}^{2}}{\alpha_{F}}\right)
\end{aligned}
$$

where $s_{F}$ and $s_{L}$ are the speed of follower and leader vehicles, and $\alpha_{F}$ and $\alpha_{L}$ are the maximum deceleration of follower and leader vehicles. The avoidance time $\tau_{1}$ is calculated using Equation (7):

$$
\tau_{1}= \begin{cases}\ddot{\tau}_{1} & \text { if } \dot{\tau}_{1}<0 \\ \min \left(\dot{\tau}_{1}, \ddot{\tau}_{1}\right) & \text { otherwise }\end{cases}
$$

Opposite: This is a case where a vehicle is heading toward another vehicle and there is a possibility of a collision. This case applies if vehicles $A$ and $B$ are moving in the opposite direction and have overlapping paths. Similar to the previous case, the net distance $d_{t}$ is calculated using Equation (4). The avoidance time $\tau_{2}$ is calculated using Equation (8):

$$
\tau_{2}=\frac{d_{t}-\frac{s_{A}^{2}}{2 \alpha_{A}}-\frac{s_{B}^{2}}{2 \alpha_{B}}}{s_{A}+s_{B}}
$$

Intersection: This is a case where two vehicles have intersecting paths and therefore there is a possibility of a collision. This case applies if trajectory lines of vehicles $A$ and $B$ intersect each other. This case covers any other conditions besides the previous two cases. Given the two trajectory lines of the vehicles, an intersection point $C\left(x_{C}\right.$, $\left.y_{C}\right)$ can be computed using simple geometry calculations. Using the intersection point, the expected time-to-intersection for both vehicles $t_{A C}$ and $t_{B C}$ can be calculated using the following formulas:

$$
\begin{aligned}
& t_{A C}=\frac{d_{A C}}{s_{A}} \operatorname{sign}\left(\overrightarrow{A C} \cdot\left[\sin \theta_{A}, \cos \theta_{A}\right]\right) \\
& t_{B C}=\frac{d_{B C}}{s_{B}} \operatorname{sign}\left(\overrightarrow{B C} \cdot\left[\sin \theta_{B}, \cos \theta_{B}\right]\right) \\
& d_{A C}=|\overrightarrow{A C}|-\frac{1}{2}\left(\frac{w_{B}}{\sin \theta_{C}}+\frac{w_{A}}{\tan \theta_{C}}\right) \\
& d_{B C}=|\overrightarrow{B C}|-\frac{1}{2}\left(\frac{w_{A}}{\sin \theta_{C}}+\frac{w_{B}}{\tan \theta_{C}}\right)
\end{aligned}
$$

where $s_{A}$ and $s_{B}$ are the speed of vehicles $A$ and $B$, respectively, $A C$ is a vector from point $A$ to point $C, B C$ is a vector from point $B$ to point $C$, and $\operatorname{sign}()$ is a sign function to identify if a vehicle has passed through the intersection. A route contention exists if both vehicles are expected to arrive at the intersection point around the same time. This can be determined by defining a time frame for each vehicle $t_{A}$ and $t_{B}$, where $t_{A C} \leq t_{A} \leq\left(t_{A C}+c_{A}\right)$ and $t_{B C} \leq t_{B} \leq\left(t_{B C}+c_{B}\right)$, such that $t_{A} \cap t_{B} \neq \varnothing$ signifies a route contention. The contention time windows $c_{A}$ and $c_{B}$ are determined by considering the intersection angle $\theta_{C}=$ $\angle A C B$ and each vehicle size and speed.

$$
\begin{aligned}
& \tilde{C}_{A}=\frac{1}{s_{A}}\left(\frac{w_{B}}{\sin \theta_{C}}+\frac{w_{A}}{\tan \theta_{C}}+l_{A}\right) \\
& \tilde{c}_{B}=\frac{1}{S_{B}}\left(\frac{w_{A}}{\sin \theta_{C}}+\frac{w_{B}}{\tan \theta_{C}}+l_{B}\right)
\end{aligned}
$$

If there is a route contention then the avoidance times $\tau_{3 A}$ and $\tau_{3 B}$ are calculated using the following equations:

$$
\tau_{3 A}=t_{A C}-\frac{s_{A}}{2 \alpha_{A}} \quad \tau_{3 B}=t_{B C}-\frac{s_{B}}{2 \alpha_{B}}
$$

\subsection{Determining Danger Severity}

Since a vehicle can endanger more than one other vehicle, the beaconing interval should be adjusted according to the interaction that has the highest danger severity. Given the interaction graph $G=(V, E)$, the maximum danger severity of a vehicle $v_{i} \in V$ can be obtained from the interaction graph by finding the highest weight $\omega_{i j}$ from all of the outgoing edges of $v_{i}$ : 


$$
\omega_{\max }\left(v_{i}\right)=\max _{\left\langle v_{i}, v_{j}\right\rangle \in E}\left(\omega_{i j}\right)
$$

Using $\omega_{\max }$, each subject vehicle calculates the sum of maximum weight $\tilde{\omega}$ :

$$
\tilde{\omega}=\sum_{v \in V} \omega_{\max }(v)
$$

The sum of maximum weight $\tilde{\omega}$ reflects a temporary local knowledge of the beaconing load within the radio range of the subject vehicle. If a vehicle knows the value of $\tilde{\omega}$ in its neighboring area, it can estimate the beaconing rate of other neighboring vehicles, which is equivalent to the beaconing load. The value of $\tilde{\omega}$ of each subject vehicle is included in every beacon message sent. Hence, each vehicle can obtain the sum of $\tilde{\omega}$ for all its neighboring vehicles, defined as $\tilde{\omega}_{v}, v \in V$. The total sum of danger severity $\omega_{\max }$ in its neighboring area is calculated by finding the largest $\tilde{\omega}_{v}$ :

$$
\tilde{\omega}_{\max }=\max _{v \in V}\left(\tilde{\omega}_{v}\right)
$$

\subsection{Rate-Adaptive Beaconing Protocol}

The proposed concept of rate adaptation scheme has been developed and implemented as a Context-aware Adaptive Rate (CAR) beaconing protocol. Algorithm 1 describes the CAR protocol, which in principle works as follows:

Algorithm 1. Context-aware adaptive rate protocol.

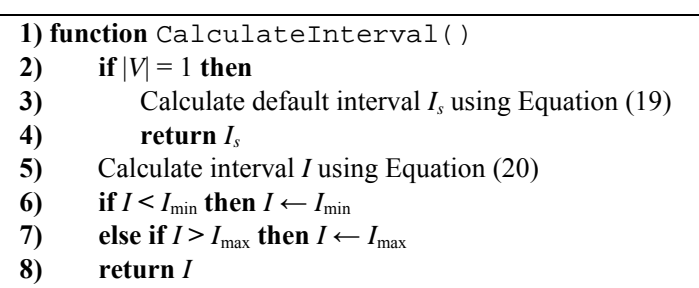

9) procedure SendMessage ( )

10) Get the vehicle self state $v$ from the positioning system

11) Update $(G, v)$

12) Create a new beacon message $m$ that contains the current self state

13) Transmit $m$ using WSMP

14) $t_{\text {prev }} \leftarrow t_{\text {now }}$

15) $I_{\text {new }} \leftarrow$ CalculateInterval( )

16) Execute SendMessage ( ) after interval $I_{\text {new }}$

17) procedure ReceiveMessage $(\mathrm{m})$

18) Retrieve the vehicle state $v_{i}$ from $m$

19) Update $\left(G, v_{i}\right)$

20) if $t_{\text {prev }}$ is defined then

21) Cancel any scheduled transmission

22) $\quad I_{\text {new }} \leftarrow$ CalculateInterval( )

23) $\quad I_{\text {now }} \leftarrow t_{\text {now }}-t_{\text {prev }}$

24) if $I_{\text {now }}<I_{\text {new }}$ then

25) Execute SendMessage ( ) after interval $\left(I_{\text {new }}-I_{\text {now }}\right)$ 26) else

27) SendMessage()
1) When a vehicle receives a beacon message from another vehicle, information from the message is used to update the interaction graph, which is locally maintained by the vehicle. Two different vehicles may not have the same information in their interaction graphs. However, closely spaced vehicles will most likely have similar information.

2) A vehicle sends a beacon message repeatedly with a dynamic interval, which is calculated using a function that utilizes the interaction graph. Whenever the interacttion graph is updated or modified, the interval is also readjusted.

The minimum interval $I_{\min }$ is the lower bound that is used to limit the beaconing interval to the smallest reasonable value. For example, a vehicle at speed $126 \mathrm{~km} / \mathrm{h}$ $(35 \mathrm{~m} / \mathrm{s})$ can travel $1.75 \mathrm{~m}$ within an interval of $50 \mathrm{~ms}$. This means that the $50 \mathrm{~ms}$ interval is small enough to give a reasonable distance error of less than two meters. The maximum interval $I_{\max }$ is the upper bound that is used to limit the interval to the largest reasonable value. For example, the $I_{\max }$ parameter can be set to one second to ensure that a beacon message is always sent at least one every second. A time $t_{\text {now }}$ is the present or most current time given by the system clock. The system clock can be globally synchronized by using the GPS.

The CalculateInterval() function calculates the beaconing interval based on the danger severity of the current road traffic situation. If a vehicle has no neighboring vehicle, which means that there are no other vehicles within its communication range, this function returns a default interval $I^{\prime}$. The default interval is calculated based on the vehicle speed using Equation (19):

$$
I^{\prime} \begin{cases}\frac{e_{t}}{s} & \text { if } s>0 \text { and } \frac{e_{t}}{s}<I_{\max } \\ I_{\min } & \text { if } \frac{e_{t}}{s}<I_{\min } \\ I_{\max } & \text { otherwise }\end{cases}
$$

where $s$ is vehicle current speed and $e_{t}$ is an error tolerance threshold. A higher speed will result in a smaller interval to keep a possible distance error less than the threshold $e_{t}$. The threshold $e_{t}$ is a parameter that can be set based on an assumption of acceptable position or distance error in the CCWS. If a vehicle has one or more neighboring vehicles, this function returns the interval $I$ calculated using Equation (20):

$$
I=\frac{1}{\frac{1}{I_{\max }}+\frac{\omega_{\max }}{\tilde{\omega}_{\max }}\left(\frac{\lambda_{\max }}{T}-\frac{|V|}{I_{\max }}\right)}
$$

The formula calculates an interval proportionally based on a vehicle's danger severity $\omega_{\max }$ and the sum of neighboring vehicles' danger severity $\omega_{\max }$, in which the resulting channel load is restricted to the maximum beaconing load $\lambda_{\max }$. The resulting interval $I$ is bounded to 
the minimum and maximum interval such that $I_{\min } \leq I \leq$ $I_{\max }$.

A vehicle $v$ starts sending beacon messages after its engine has been started. A beacon message is transmitted by invoking the SendMessage ( ) procedure. This procedure first acquires current vehicle self state, such as position, speed, and heading, from the positioning system. The Update $(G, v)$ procedure updates and recalculates the interaction graph $G$ with the new information. A new data packet that encodes the state information is created and transmitted using WAVE Short Message Protocol (WSMP) as defined in the IEEE 1609.3 standard [5]. The time of transmission is kept in $t_{\text {prev }}$. The next beacon transmission is then scheduled by executing the Send Message() procedure after an interval calculated by the CalculateInterval() function.

The ReceiveMessage () procedure is called when a vehicle $v$ receives a beacon message $m$ from another vehicle $v_{i}$. This procedure decodes the state of vehicle $v_{i}$ from $m$ and updates the interaction graph $G$ of vehicle $v$ with the new information. Every time a beacon message is received, it is likely that the interactions among neighboring vehicles have changed. Therefore, any scheduled beacon transmission is canceled and the next transmission is scheduled with a new interval. The new interval $I_{\text {new }}$ is calculated using the CalculateInterval() function. The actual current interval $I_{\text {now }}$ is the duration elapsed since the last transmission time $t_{\text {prev }}$ until the current time $t_{\text {now. }}$. If the new interval is longer than the actual current interval, the next beacon transmission is then scheduled at time $I_{\text {new }}-I_{\text {now }}$. Otherwise, a beacon message must be sent immediately by invoking the SendMessage( ) procedure.

\section{Evaluation}

The performance of the Context-aware Adaptive Rate (CAR) scheme is evaluated by performing an integrated simulation of a vehicular wireless network, vehicles moving on a straight road with multiple lanes, and collisions between vehicles caused by unsafe situations. The simulation program is implemented by extending the ns-3 network simulator (version 3.8) [27].

The performance of the CAR scheme is compared with several Constant Rate (CR) schemes and an existing Probabilistic Adaptive Rate (PAR) scheme [12]. In the CR schemes, beacon messages are periodically sent at a constant interval. Four different intervals were selected and represented by the CR-50 (50 ms interval), CR-100 (100 ms interval), CR-200 (200 ms interval), and CR-500 (500 ms interval) schemes. The PAR scheme is a relatively recent adaptive beaconing scheme that improves tracking accuracy under various traffic conditions by calculating transmission probability based on suspected tracking error on neighboring vehicles. To clearly demonstrate the benefits of the new context-aware adaptive technique, all the compared schemes are implemented without using any kind of position prediction model.

\subsection{Performance Metrics}

We evaluate the safety and communication performances in terms of efficiency and scalability. A scheme is efficient if it generates less network load to maintain a certain safety level. A scheme is scalable if it is able to maintain safety and communication performances in various traffic scenarios with different density.

The aim of the CCWS is to improve road safety by preventing vehicle collisions caused by the error or limited perception of human drivers. Therefore, the number of vehicle collisions is used as the metric to assess the safety performance (as in [28]). A beaconing scheme has a better safety performance if using the scheme results in a smaller number of potential vehicle collisions. The number of potential vehicle collisions is measured by simulating an accident scenario on a typical highway. To study the effect of different beaconing schemes on the number of potential collisions, the simulation is designed in such a way so that a collision will occur only if a beacon message is not received in time.

The communication performance involves the metrics of dissemination latency or delay, actual channel usage, and probability of message reception. The latency is the duration between the time when a beacon message is sent to the MAC layer and the time when it is received by other vehicles. A lower latency gives a better chance for a vehicle to avoid a collision. The actual channel usage is measured by averaging channel busy time from all nodes during the simulation time. As such, the measured usage includes the PHY and MAC protocols overhead. Higher channel usage increases the possibility of channel congestion. The probability of message reception is the probability that a beacon message is successfully received by a node located at a particular distance from a sender node. Higher probability of message reception indicates fewer packet collisions.

A good overall performance is indicated by both safety and communication performance. This means that a good scheme must achieve a low number of collisions, low latency, low channel usage, and high probability of message reception. However, emphasize is given to the number of potential collisions metric since the ultimate goal of the CCWS is to improve safety.

\subsection{Simulation Design and Setup}

\subsubsection{Wireless Communication}

Each vehicle repeatedly sends beacon messages during the simulation duration at an interval determined by the beaconing schemes. For example, the CR-100 scheme 
sends a beacon message every 100 milliseconds. The beacon message size is set to a constant value of 500 bytes, excluding the MAC protocol specific header. A constant message size is used to provide a consistent comparison result. The transmission power is configured to $19 \mathrm{dBm}$. The probabilistic Nakagami distribution is selected for the radio propagation loss model, as field tests on highways showed that the Nakagami distribution is suitable to be used on vehicular communication in highway scenarios [19]. The parameter of $m=1$ is set to simulate severe fading conditions; therefore, demonstrating the beaconing performance in the worst case scenario.

The parameters for PHY and MAC protocols are set according to the IEEE $802.11 \mathrm{p}$ draft standard, which operates at $5.9 \mathrm{GHz}$ on a $10 \mathrm{MHz}$ control channel $(\mathrm{CCH})$. The PHY data rate is configured to $6 \mathrm{Mbps}$, which is the optimal value for safety communication [29]. The channel switching scheme is currently not implemented, so the CCWS applications can utilize the entire $10 \mathrm{MHz}$ $\mathrm{CCH}$ bandwidth. The MAC layer is configured to ad hoc mode with QoS support using the EDCA mechanism as described in IEEE 802.11e. The priority for beacon messages is set to AC_VI (second highest). All beaconing schemes are implemented as application level protocols in the simulator that use the IEEE 1609 WAVE Short Message Protocol (WSMP) [5]. Common configuration parameters related to communication are summarized in Table 1.

\subsubsection{Road Traffic and Accident Scenario}

The simulation of vehicles moving on a road is staged on a typical multi-lane $2 \mathrm{~km}$ highway as illustrated in Figure 3. To demonstrate the scalability of the CAR scheme, five scenarios with different average vehicle densities are evaluated: VD-30, VD-60, VD-90, VD-120, and VD-150. The vehicle density starts from 30 vehicles/km (VD-30) up to 150 vehicles/km (VD-150). Each scenario is designed with different numbers of vehicles and lanes to create a realistic situation with a desired density. Table 2 shows the parameters of the scenarios. The number of

Table 1. Common configuration parameters.

\begin{tabular}{ll}
\hline Parameter & Value \\
\hline PHY and MAC protocol & $802.11 \mathrm{p}$ \\
$802.11 \mathrm{p}$ data rate & $6 \mathrm{Mbps}$ \\
Propagation loss model 1 & Three log distance \\
Propagation loss model 2 & Nakagami \\
Transmission power & 19 dBm \\
Beacon message size & 500 bytes \\
Beacon priority level & AC_VI \\
CAR parameters & $I_{\text {min }}=50 \mathrm{~ms}, I_{\max }=1000 \mathrm{~ms}$ \\
\hline
\end{tabular}

vehicles on each lane is randomized. Vehicles on the same lane travel at the same speed, which is determined based on the vehicle density. The distance between two consecutive vehicles $d_{i, j}$ is random, but the value is ensured to be greater than the required safety distance. As such, a collision is always avoidable provided that a beacon message is received on time. For each scenario, simulations with different random seeds were performed 50 times. Each simulation instance uses a random road traffic situation (random speed and inter-vehicle distance). Results from the simulation are averaged from the 50 runs.

The simulation implements a basic CCWS function for each vehicle. If a collision is likely to occur, the CCWS warns the driver, which will then stop the vehicle to avoid the collision. To evaluate the safety performance of the CCWS, collisions between vehicles are simulated by assuming that some drivers become distracted or inattentive. A distracted driver cannot promptly react to avoid a collision with a leading vehicle, unless they are warned by the CCWS. To prevent the collision, the CCWS must warn the driver at the right time. The timing of warning is calculated based on the tracked state of neighboring vehicles. If up-to-date beacon messages were not promptly received, the warning calculation will be inaccurate, and a collision may occur accordingly. Therefore, the safety performance of different beaconing schemes can be evaluated based the number of potential collisions that cannot be prevented.

The percentage of distracted or inattentive drivers in each simulation instance is determined using a parameter. The performance of the beaconing schemes can be fully demonstrated by using a worst case scenario that assumes all the drivers are inattentive. However, to make the simulation more realistic, the number of inattentive

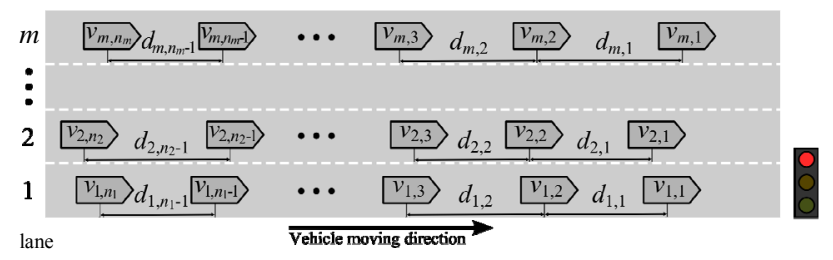

Figure 3. Illustration of the simulated highway scenarios.

Table 2. Specific parameters for scenarios with different vehicle densities.

\begin{tabular}{cccc}
\hline Scenario & $\begin{array}{c}\text { Number of } \\
\text { vehicles }\end{array}$ & $\begin{array}{c}\text { Number of } \\
\text { lanes }\end{array}$ & $\begin{array}{c}\text { Speed variation } \\
\text { range }\left(\mathrm{m} / \mathrm{s}^{2}\right)\end{array}$ \\
\hline VD-30 & 60 & 2 & $25-30$ \\
VD-60 & 120 & 3 & $15-25$ \\
VD-90 & 180 & 3 & $10-15$ \\
VD-120 & 240 & 6 & $15-25$ \\
VD-150 & 300 & 6 & $10-15$ \\
\hline
\end{tabular}


drivers is set to 15 percent of the total vehicles in each scenario. The percentage is obtained from statistics of driver inattention in the US [30], which are based on the analysis of five years of data.

The simulation models a situation when vehicles stop at a red traffic light. Each vehicle at the front end of each lane $v_{\text {laneld, } 1}$ will start decelerating normally at $4.9 \mathrm{~m} / \mathrm{s}^{2}$ when approaching the end of the road, until it stops completely right at the end of the road. To avoid a collision, a following vehicle must decelerate at the right time depending on the relative position and speed of its leading vehicle. A normal vehicle will start decelerating based on the calculation using the actual position and speed of its leading vehicle. A vehicle with a distracted driver will start decelerating only after its warning system predicts a collision based on the known (tracked) position and speed of its leading vehicle, instead of the actual position and speed. Inaccurate position and speed prediction may result in some collisions depending on the interaction between vehicles.

Common parameters related to the vehicles are given in Table 3. Driver's reaction time is set to a constant value of $1.5 \mathrm{~s}$. A minimum inter-vehicle gap of $2 \mathrm{~m}$ is used as a tolerance buffer in the calculation of collision prediction. The error tolerance threshold $e_{t}$ is set to the same value as the minimum gap. A simulation instance finishes when all vehicles stop moving.

\subsection{Simulation Results}

Since the CAR scheme is expected to perform differently given a different maximum beaconing load, the performance of CAR scheme is firstly evaluated by varying the $\lambda_{\max }$ parameter from 0.1 to 1.0 . The average results from all scenarios show that the parameter $\lambda_{\max }=1.0$ gives the least number of collisions. However, there is no signifycant difference in the number of collisions with $\lambda_{\max } \geq$ 0.6 , in which all the average collisions are below 0.2 . The distance error is measured from the simulations by accumulating the distance between the tracked position and the actual position of a vehicle every $100 \mathrm{~ms}$ and averaging the result. The results indicate that the error decreases significantly as the $\lambda_{\max }$ increases. A higher value of $\lambda_{\max }$ implies a shorter beaconing interval, which also results in a higher actual channel usage. And as expected in a wireless network that uses the CSMA MAC protocol,

Table 3. Common parameters for the highway scenario.

\begin{tabular}{lc}
\hline Parameter & Value \\
\hline Driver's reaction time & $1.5 \mathrm{~s}$ \\
Vehicle length & $4 \mathrm{~m}$ \\
Min. inter-vehicle gap & $2 \mathrm{~m}$ \\
Vehicle deceleration & $4.9 \mathrm{~m} / \mathrm{s}^{2}$ \\
Highway length & $2000 \mathrm{~m}$ \\
\hline
\end{tabular}

the overall probability of message reception decreases as the channel usage increases. Although the CAR scheme with $\lambda_{\max }=1.0$ has the lowest reception probability, it has the fewest number of collisions. From the initial evaluation, we conclude that the CAR scheme performs the best using the parameter $\lambda_{\max }=1.0$. The results also confirm the proposition that safety performance cannot be measured solely by the tracking accuracy metric or by the communication performance such as successful message reception rate.

The performance of the CAR scheme with $\lambda_{\max }=1.0$ is then compared to CR and PAR schemes. The average number of collisions resulting from the use of each scheme in each scenario is shown in Table 4. The total average of the results from all scenarios indicates the overall safety performance of each scheme. From the safety perspective, the CR-50 scheme has the worst performance in the scenario with the highest vehicle density (VD-150). Such a result indicates severe channel congestion because the channel capacity is overloaded. The CR-500 scheme has the worst performance in all other scenarios (VD-30 to VD-120), which indicates that the beaconing rate of 2 messages per second is not enough in most situations to ensure safety. The CR-100 scheme has the best average result compared to the other $\mathrm{CR}$ schemes. Therefore, further performance comparisons will only include the result from CR-100, PAR, and CAR schemes.

The CAR scheme can prevent all potential collisions in the VD-60 and VD-90 scenarios and has the lowest number of collisions in the VD-120 and VD-150 scenarios. The total average shows that the CAR scheme has the best safety performance, followed by the PAR, CR100, CR-200, CR-500, and CR-50 schemes. The average number of collisions for the CAR scheme in every scenario is always less than 0.23 , which demonstrates that it can ensure safety in various traffic situations.

Figure 4 plots the percentage of occurred collisions calculated by normalizing the number of collisions to the maximum number of possible collisions. The result shows the magnitude of safety improvement that the CAR scheme is able to achieve in comparison to the CR-100 and PAR schemes. The average latency of one hop transmissions is shown in Figure 5. The latencies for

Table 4. Number of vehicle collisions in different scenarios.

\begin{tabular}{crrrrrr}
\hline Scenario & CR-50 & CR-100 & CR-200 & CR-500 & PAR & CAR \\
\hline VD-30 & 0.00 & 0.10 & 0.34 & 3.16 & 0.02 & 0.02 \\
VD-60 & 0.20 & 0.16 & 0.74 & 4.34 & 0.00 & 0.00 \\
VD-90 & 0.24 & 0.04 & 0.34 & 2.38 & 0.08 & 0.00 \\
VD-120 & 3.04 & 1.50 & 1.78 & 9.78 & 0.54 & 0.20 \\
VD-150 & 25.34 & 1.22 & 1.68 & 8.44 & 0.66 & 0.22 \\
Average & 5.764 & 0.604 & 0.976 & 5.620 & 0.260 & 0.088 \\
\hline
\end{tabular}




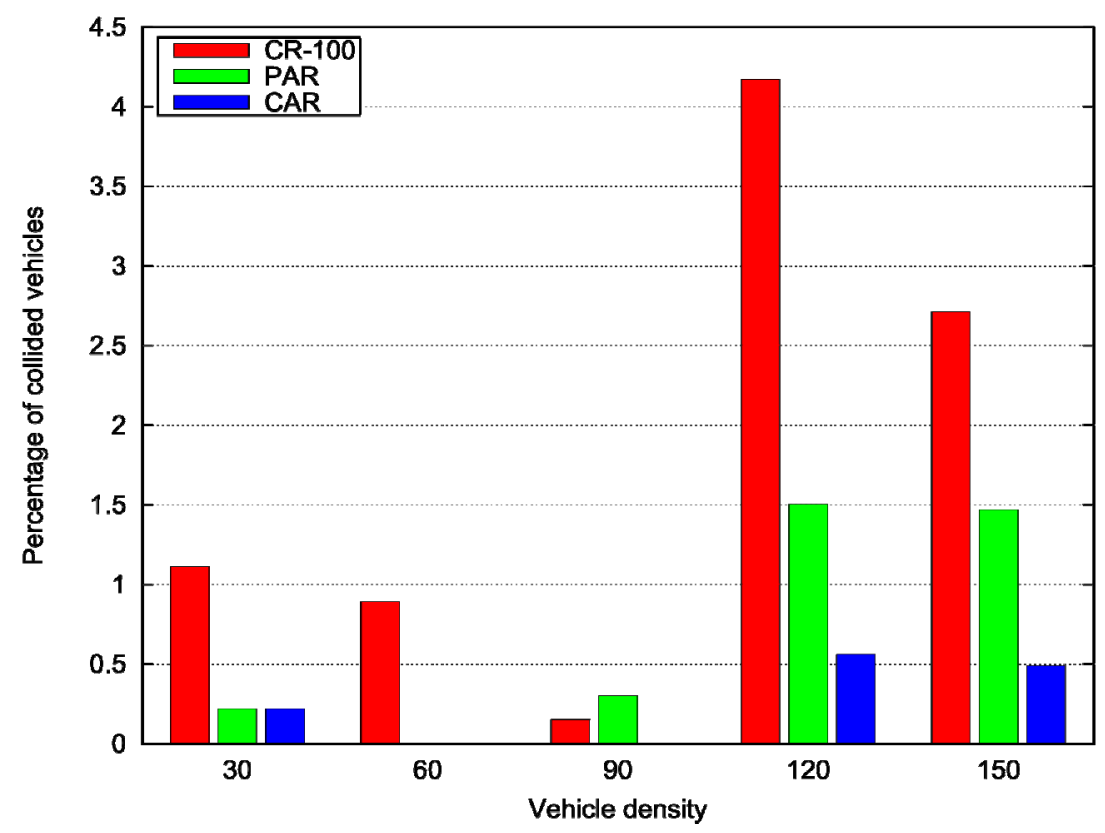

Figure 4. Percentage of occurred collisions.

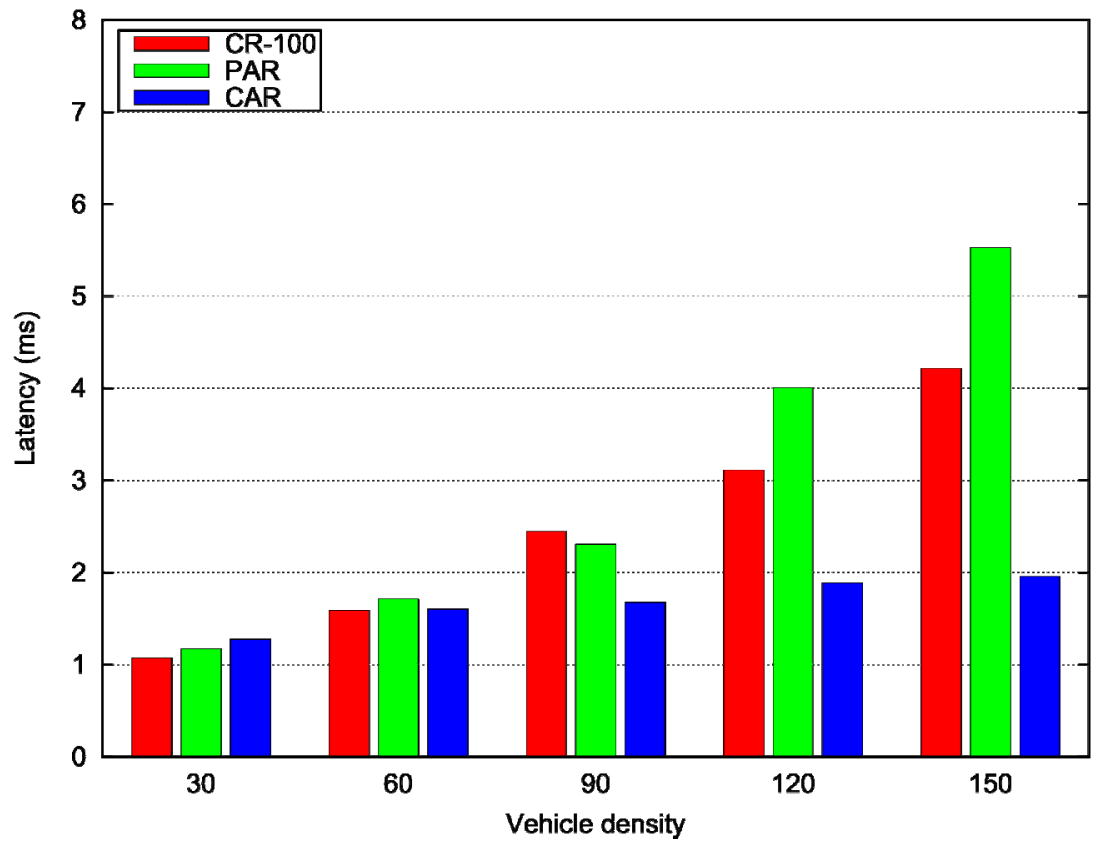

Figure 5. Latency of one hop transmissions.

all the compared schemes are all below $6 \mathrm{~ms}$, which make their differences relatively insignificant. However, CAR scheme can maintain the latency below $2 \mathrm{~ms}$ in all scenarios. Average channel usage during the simulation duration is shown in Figure 6. It demonstrates the efficiency of the CAR scheme compared to the CR-100 scheme in most scenarios. The PAR scheme has the lowest channel usage, but it generates more vehicle collisions compared to the CAR scheme. Both the PAR and CAR schemes are more scalable because they can main- tain channel usage below $65 \%$ even in high density scenarios.

To evaluate the communication reliability, Figure 7 compares the probability of message reception between the CR-100, PAR, and CAR schemes. The probability is plotted with respect to the distance between a receiver and a sender. Figures 7(a) and (b) show that the reliability of the CR-100 and PAR schemes decreases significantly when the vehicle density increases. In contrast, Figure 7(c) shows that the reliability of the CAR scheme 
does not change significantly with different vehicle densities. In the VD-30 scenario, the overall probabilities of message reception of the CR-100 and CAR schemes are relatively similar. However, the resulting number of collisions in the same scenario for the CAR scheme is smaller than for the CR-100 scheme because the CAR scheme is able to prioritize vehicles in the most danger.

The results of communication performance show that, in general, a higher channel usage causes a higher latency and a lower probability of message reception. The CAR scheme can limit its channel usage and keep the probability of message reception within acceptable levels in any scenario with different vehicle densities. Although the CAR scheme cannot achieve a very high probability of message reception, its safety performance is the best.

\section{Discussion}

Simulation results demonstrate the safety, efficiency, and scalability of the proposed CAR scheme. In terms of safety, the CAR scheme constantly performs better than the other schemes for all tested scenarios with different vehicle densities, as indicated by the average of the vehicle collisions. Our experiments show that the CR-100 scheme has the best overall performance among the constant rate schemes. It seems that the popular assumption of using an interval of $100 \mathrm{~ms}$ for beaconing [8,19,31] may not be without grounds. As expected, the adaptive schemes (CAR and PAR) perform better than all the constant rate schemes because the adaptive schemes are able to control channel congestion. CAR and PAR schemes have the same safety performance in low density scenarios (VD-30 and VD-60). However, CAR scheme significantly outperforms PAR scheme in high density scenarios because of the prioritization strategy. This shows that prioritizing vehicles based on their danger severity can improve safety.

In terms of efficiency, the CAR scheme can maintain its actual channel usage between $45 \%$ and $65 \%$ of the capacity in all the scenarios. It is better than the CR-100 schemes that can utilize almost $90 \%$ of channel capacity in high density scenarios, but with a lower safety performance. Safety performance of the CR-100 scheme is the lowest in the VD-120 and VD-150 scenarios because of the high channel usage. It is clear that using a constant rate scheme may cause channel congestion that can significantly reduce safety, particularly when road traffic becomes denser such as in a traffic jam. Although the PAR scheme utilizes the least channel capacity, the PAR safety performance is lower than for the CAR. The result shows that the best safety performance is not achievable by only reducing channel usage without prioritizing vehicles in the most danger. The result confirms that the CAR scheme is able to achieve its objective, which is to improve both efficiency and safety.

The safety and communication performances of the CAR scheme are more scalable than those of the CR-100 and PAR schemes in all tested scenarios, as indicated by the vehicle collisions, latency, and probability of message reception. In low density scenarios, all the schemes perform relatively well because the channel capacity is still sufficient. In high density scenarios, the CAR scheme

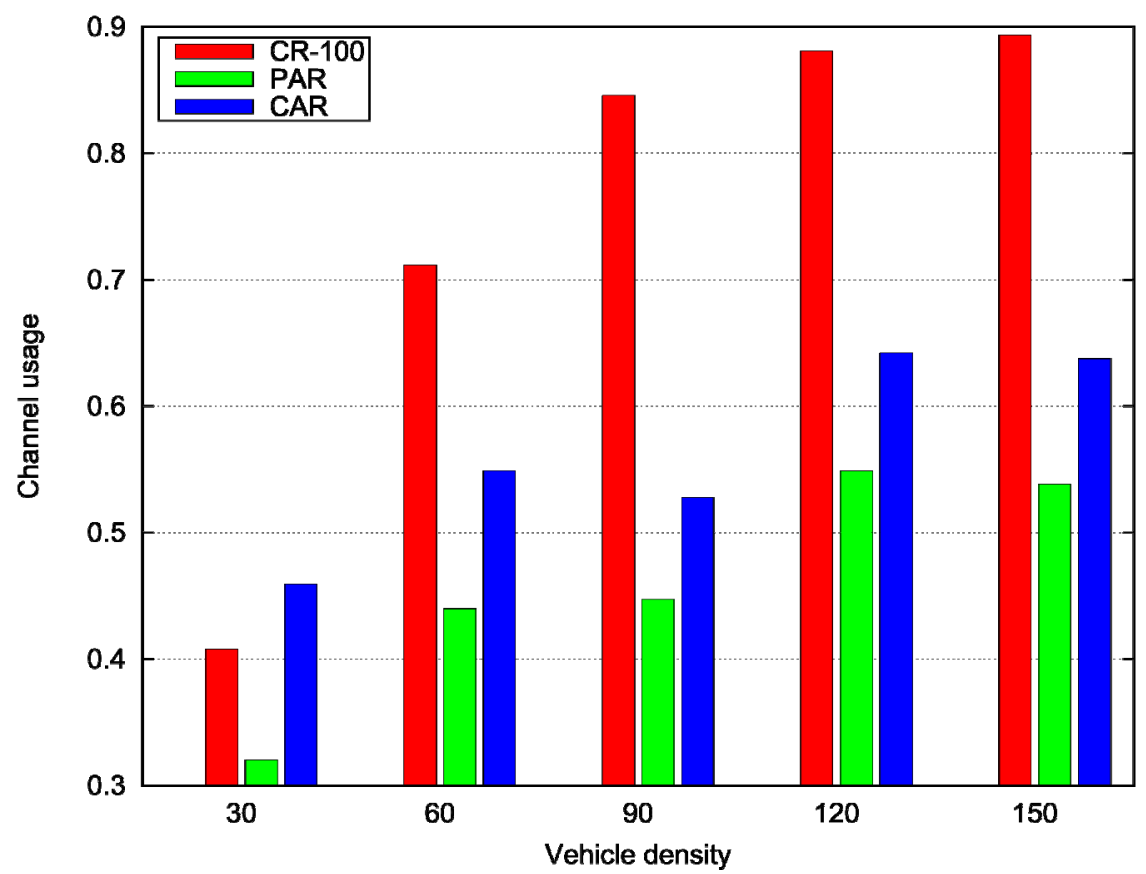

Figure 6. Channel usage during the simulation duration. 


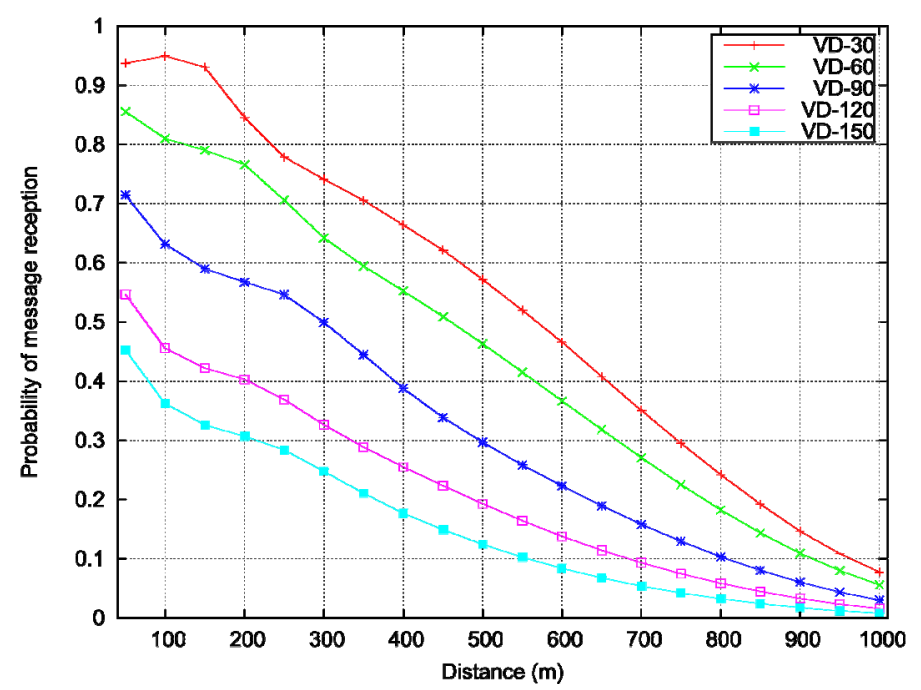

(a)

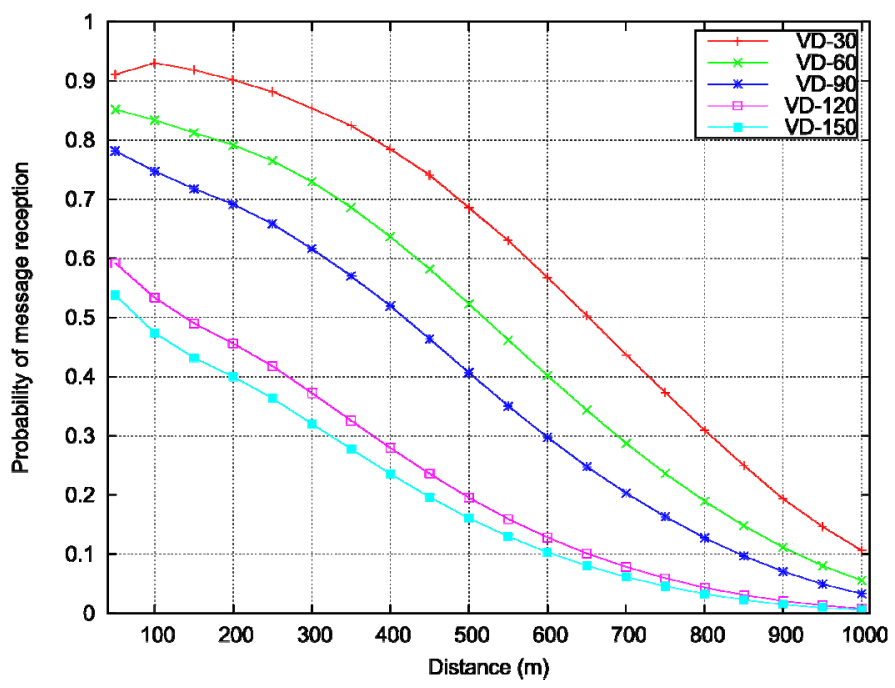

(b)

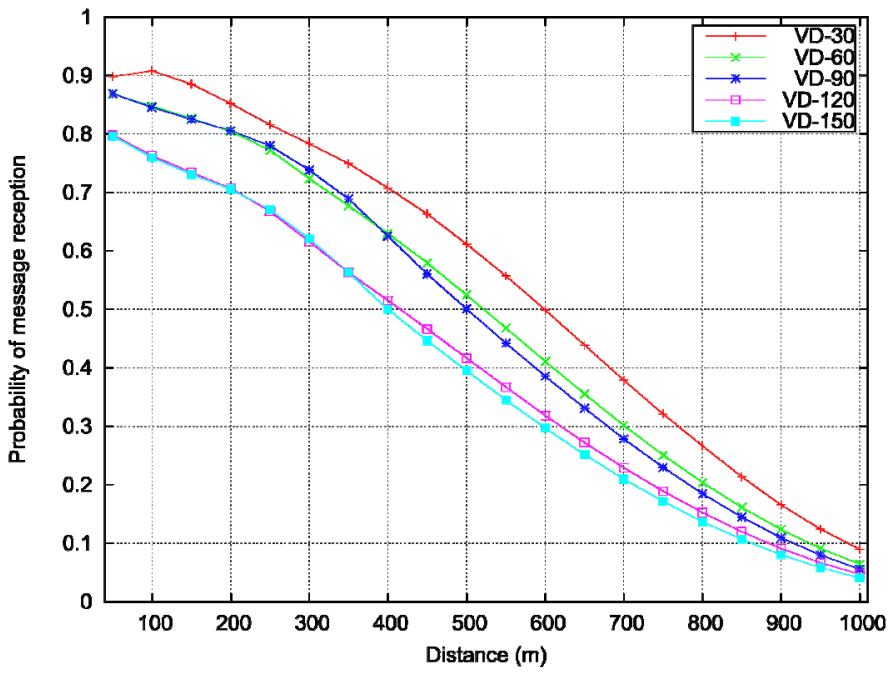

(c)

Figure 7. Probability of message reception with respect to the distance from the sender. (a) CR-100 scheme; (b) PAR scheme; (c) CAR scheme with $\lambda_{\max }=1.0$. 
significantly outperforms all the other schemes. The average number of collisions indicates the safety performance for all the scenarios. The CAR scheme comes with the smallest average number of collisions of 0.088 , followed by the PAR scheme with an average number of collisions of 0.260 , which is almost three times larger than the CAR's result. The CAR scheme has the best safety performance in almost all scenarios. Figure 5 shows that the latency for the CAR scheme is kept at below $2 \mathrm{~ms}$ in all scenarios while the latency for the CR-100 and PAR schemes can exceed $3 \mathrm{~ms}$ in some scenarios. The CAR scheme can ensure a more stable and relatively high probability of message reception in all scenarios. The result demonstrates the scalability of the CAR scheme to ensure the CCWS safety and communication performances under various road traffic conditions.

It is expected that the maximum beaconing load parameter $\lambda_{\max }$ cannot control the channel usage precisely because each vehicle only relies on its own local one-hop knowledge. For $\lambda_{\max } \leq 0.3$, the actual channel usage is slightly more than the specified limit. For $\lambda_{\max }>0.3$, the actual channel usage is getting much lower than the specified limit as its value increases. The discrepancy is reasonable because the parameter $\lambda_{\max }$ is used just as a maximum limit of the channel usage estimation. Since the beaconing interval is bounded between $50 \mathrm{~ms}$ and $1000 \mathrm{~ms}$, the maximum limit may not be reached in some situations, such as when vehicles are not moving. The objective of the CAR scheme is not about precise control of the actual channel usage.

\section{Conclusions}

In this article, we presented a new context-aware adaptive beaconing rate scheme to improve the performance of vehicular safety communication. The original contribution of this research is a new method to adapt the beaconing rate dynamically to the context, which includes the estimated channel load and the danger severity. The proposed scheme estimates the danger severity of each vehicle by using the interaction graph model. Vehicles with the highest danger severity are facing the highest risk of collision, and therefore must be prioritized. The beacon messages are sent at a shorter interval for these vehicles to increase their chance to avoid a possible collision.

Simulation results have demonstrated that the proposed scheme outperforms both the existing adaptive rate and non-adaptive rate schemes in terms of the efficiency, scalability, and safety. The proposed scheme is able to reduce the potential collision rate significantly, and therefore improve safety. Efficiency is demonstrated by having a lower channel usage compared to the existing schemes of a similar safety performance. Scalability is demonstrated by having a relatively consistent safety and communication performance across various scenarios with different vehicle densities.

The context-aware adaptive scheme can be extended by incorporating existing ideas and concepts to improve the beaconing performance. In future work, we will study the benefits of combining our proposed scheme with a prediction scheme that uses a threshold policy to further reduce the beaconing rate and an aggregation or piggybacking scheme to improve the successful message reception rate. To further improve the beaconing efficiency, the next step would be to investigate an extended scheme that adapts both the repetition interval and the transmission power.

\section{Acknowledgements}

This research was supported by Queensland University of Technology (QUT) and the Commonwealth of Australia, through the Cooperative Research Center for Advanced Automotive Technology (AutoCRC). Computational resources and services used in this work were provided by the High Performance Computing and Research Support Unit, QUT, Brisbane, Australia.

\section{REFERENCES}

[1] H. S. Tan and J. Huang, "DGPS-Based Vehicle-to-Vehicle Cooperative Collision Warning: Engineering Feasibility Viewpoints," IEEE Transactions on Intelligent Transportation System, Vol. 7, No. 4, 2006, pp. 415-428. doi:10.1109/TITS.2006.883938

[2] R. Sengupta, S. Rezaei, S. E. Shladover, D. Cody, S. Dickey and H. Krishnan, "Cooperative Collision Warning Systems: Concept Definition and Experimental Implementation," Journal of Intelligent Transportation Systems, Vol. 11, No. 3, 2007, pp. 143-155. doi:10.1080/15472450701410452

[3] G. K. Mitropoulos, I. S. Karanasiou, A. Hinsberger, F. Aguado-Agelet, H. Wieker, H.-J. Hilt, S. Mammar and G. Noecker, "Wireless Local Danger Warning: Cooperative Foresighted Driving Using Intervehicle Communication," IEEE Transactions on Intelligent Transportation Systems, Vol. 11, No. 3, 2010, pp. 539-553. doi:10.1109/TITS.2009.2034839

[4] "IEEE 802.11 Standard Amendment 6: Wireless Access in Vehicular Environments," IEEE Std. 802.11p, 2010.

[5] "IEEE Standard for Wireless Access in Vehicular Environments (WAVE)-Networking Services," IEEE Std. 1609.3, 2010, pp. 1-144.

[6] J. Yin, T. ElBatt, G. Yeung, B. Ryu, S. Habermas, H. Krishnan and T. Talty, "Performance Evaluation of Safety Applications over DSRC Vehicular Ad Hoc Networks," Proceedings of the 1st ACM International Workshop on VANET, Philadelphia, 1 October 2004.

[7] P. Alexander, D. Haley and A. Grant, "Cooperative Intelligent Transport Systems: 5.9-Ghz Field Trials," Proceedings of the IEEE, Vol. 99, No. 7, 2011, pp. 12131235. doi:10.1109/JPROC.2011.2105230 
[8] The CAMP Vehicle Safety Communications Consortium, "Vehicle Safety Communications Project-Task 3 Final Report-Identify Intelligent Vehicle Safety Applications Enabled by DSRC," Tech. Rep. DOT HS 809859 , NHTSA, US Department of Transportation, Washington, 2005.

[9] S. Yousefi, M. Fathy and A. Benslimane, "Performance of Beacon Safety Message Dissemination in Vehicular Ad Hoc Networks (VANETs)," Journal of Zhejiang University-Science A, Vol. 8, No. 12, 2007, pp. 1990-2004.

[10] M. van Eenennaam, W. K. Wolterink, G. Karagiannis and G. Heijenk, "Exploring the Solution Space of Beaconing in VANETs," Proceedings of the IEEE Vehicular Networking Conference, Tokyo, 28-30 October s2009, pp. 1-8. doi:10.1109/VNC.2009.5416370

[11] M. Saito, J. Tsukamoto, T. Umedu and T. Higashino, "Design and Evaluation of Intervehicle Dissemination Protocol for Propagation of Preceding Traffic Information," IEEE Transactions on Intelligent Transportation Systems, Vol. 8, No. 3, 2007, pp. 379-390. doi:10.1109/TITS.2007.902650

[12] C.-L. Huang, Y. P. Fallah, R. Sengupta and H. Krishnan, "Adaptive Intervehicle Communication Control for Cooperative Safety Systems," IEEE Network, Vol. 24, No. 1, 2010, pp. 6-13. doi:10.1109/MNET.2010.5395777

[13] R. K. Schmidt, T. Leinmüller, E. Schoch, F. Kargl and G. Schäfer, "Exploration of Adaptive Beaconing for Efficient Intervehicle Safety Communication," IEEE Network, Vol. 24, No. 1, 2010, pp. 14-19. doi:10.1109/MNET.2010.5395778

[14] M. van Eenennaam, G. Karagiannis and G. Heijenk, "Towards Scalable Beaconing in VANETs," Proceedings of the 4th ERCIM Workshop on eMobility, Luleå, 31 May 2010, pp. 103-108.

[15] D. Jiang, V. Taliwal, A. Meier, W. Holfelder and R. Herrtwich, "Design of 5.9 Ghz DSRC-Based Vehicular Safety Communication," IEEE Wireless Communications, Vol. 13, No. 5, 2006, pp. 36-43. doi:10.1109/WC-M.2006.250356

[16] F. Schmidt-Eisenlohr, M. Torrent-Moreno, J. Mittag and H. Hartenstein, "Simulation Platform for Inter-Vehicle Communications and Analysis of Periodic Information Exchange," Proceedings of the 4th Annual Conference on Wireless on Demand Network Systems and Services, Obergurgl, 24-26 January 2007, pp. 50-58. doi:10.1109/WONS.2007.340475

[17] C. L. Robinson, D. Caveney, L. Caminiti, G. Baliga, K. Laberteaux and P. R. Kumar, "Efficient Message Composition and Coding for Cooperative Vehicular Safety Applications," IEEE Transactions on Vehicular Technology, Vol. 56, No. 6, 2007, pp. 3244-3255. doi:10.1109/TVT.2007.907325

[18] J. Mittag, F. Schmidt-Eisenlohr, M. Killat, J. Härri and H. Hartenstein, "Analysis and Design of Effective and LowOverhead Transmission Power Control for VANETs," Proceedings of the 5th ACM International Workshop on VANET, San Francisco, 14-19 September 2008. doi:10.1145/1410043.1410051

[19] M. Torrent-Moreno, J. Mittag, P. Santi and H. Hartenstein,
"Vehicle-to-Vehicle Communication: Fair Transmit Power Control for Safety-Critical Information," IEEE Transactions on Vehicular Technology, Vol. 58, No. 7, 2009, pp. 3684-3703. doi:10.1109/TVT.2009.2017545

[20] S. Rezaei, R. Sengupta, H. Krishnan, X. Guan and R. Bhatia, "Tracking the Position of Neighboring Vehicles Using Wireless Communications," Transportation Research Part C: Emerging Technologies, Vol. 18, No. 3, 2009, pp. 335-350. doi:10.1016/j.trc.2009.05.010

[21] M. Armaghan, M. Fathy and S. Yousefi, "Improving the Performance of Beacon Safety Message Dissemination in Vehicular Networks Using Kalman Filter Estimation," In: D. Slezak, et al., Eds., Communication and Networking, Springer, Berlin Heidelberg, 2009, pp. 74-82. doi:10.1007/978-3-642-10844-0 10

[22] C.-L. Huang, Y. P. Fallah, R. Sengupta, and H. Krishnan, "Information Dissemination Control for Cooperative Active Safety Applications in Vehicular Ad-Hoc Networks," Proceedings of the IEEE Global Telecommunications Conference, Honolulu, 30 November-4 December 2009, pp. 1-6.

[23] A. Sebastian, M. Tang, Y. Feng and M. Looi, "MultiVehicles Interaction Graph Model for Cooperative Collision Warning System," Proceedings of the IEEE Intelligent Vehicles Symposium, Xi'an, 3-5 June 2009.

[24] J. Huang and H.-S. Tan, "Impact of Communication Reliability on a Cooperative Collision Warning System," Proceedings of the IEEE Intelligent Transportation Systems Conference, Seattle, 30 September-3 October 2007, pp. 355-360. doi:10.1109/ITSC.2007.4357731

[25] N. M. Drawil and O. Basir, "Intervehicle-CommunicationAssisted Localization," IEEE Transactions on Intelligent Transportation Systems, Vol. 11, No. 3, 2010, pp. 678691. doi:10.1109/TITS.2010.2048562

[26] M. Green, “'How Long Does It Take to Stop?' Methodological Analysis of Driver Perception-Brake Times," Transportation Human Factors, Vol. 2, No. 2, 2000, pp. 195-216. doi:10.1207/STHF0203_1

[27] “The ns-3 Network Simulator," 2011. http://www.nsnam.org

[28] S. Biswas, R. Tatchikou and F. Dion, "Vehicle-to-Vehicle Wireless Communication Protocols for Enhancing Highway Traffic Safety," IEEE Communications Magazine, Vol. 44, No. 1, 2006, pp. 74-82. doi:10.1109/MCOM.2006.1580935

[29] D. Jiang, Q. Chen and L. Delgrossi, "Optimal Data Rate Selection for Vehicle Safety Communications," Proceedings of the 5th ACM International Workshop on VANET, San Francisco, 14-19 September 2008, pp. 30-38.

[30] J. C. Stutts, D. W. Reinfurt, L. Staplin and E. A. Rodgman, "The Role of Driver Distraction in Traffic Crashes," AAA Foundation for Traffic Safety, Washington DC, 2001. http://www.aaafoundation.org/pdf/distraction.pdf

[31] I. Chisalita and N. Shahmehri, "On the Design of Safety Communication Systems for Vehicles," IEEE Transactions on Systems, Man and Cybernetics, Part A, Vol. 37, No. 6, 2007, pp. 933-945.

doi:10.1109/TSMCA.2007.897586 